\title{
Modelling the allocation of paediatric intensive care retrieval teams in England and
}

\section{Wales}

Madeline King ${ }^{1,2}$, Padmanabhan Ramnarayan ${ }^{3}$, Sarah Emma Seaton ${ }^{4}$, Christina Pagel ${ }^{1}$, on behalf of the DEPICT Study Group

1. Clinical Operational Research Unit, University College London, 4 Taviton Street, London, WC1H 0BT, UK

2. Washington University, One Brookings Drive, St. Louis, MO 63130, USA

3. Children's Acute Transport Service (CATS), Great Ormond Street Hospital NHS Foundation Trust, 26-27, Boswell Street, London, WC1N 3JZ, UK

4. Department of Health Sciences, George Davies Centre, University Road, Leicester, LE1 $7 \mathrm{RH}, \mathrm{UK}$

Corresponding author: Christina Pagel, Clinical Operational Research Unit, UCL, 4 Taviton Street, WC1H 0BT, c.pagel@ucl.ac.uk, 02076794501 (we do not have a fax).

Main paper: 2720 words

Keywords: Intensive Care, Mathematical Modelling, System configuration 


\section{Abstract}

\section{Background}

Following centralisation of paediatric intensive care units (PICUs) in 1997, specialist PIC retrieval teams (PICRTs) were established to transport critically ill children from district general hospitals (DGHs). The current location and catchment area of PICRTs covering England and Wales are based on historical referral patterns. National quality standards specify that PICRTs should reach the patient bedside within 3 hours of accepting a referral.

\section{Objective}

To determine what proportion of demand for PICRT services can be reached within 3 hours and to explore the potential coverage impact of more stringent 'time to bedside' standards.

\section{Methods}

We used mathematical location-allocation methods to: (1) determine the optimal allocation of DGHs to current PICRT locations to minimise road journey time and calculated the proportion of demand reachable within 3 hours; 2 hours; 90 minutes; 75 minutes and 1 hour; (2) explore the impact of changing the number and location of PICRTs on demand coverage for the different time thresholds.

\section{Results}

For current (and optimal) location of 11 PICRTs, $98 \%$ (98\%) of demand is reachable within 3 hours; 86\% (91\%) within 2 hours; 59\% (69\%) within 90 minutes; 33\% (39\%) within 75 minutes; $20 \%$ (20\%) within 1 hour. Five hospitals were not reachable within 3 hours. For the 3-hour standard, 8 optimally located PICRT locations had similar coverage as the current 11 locations.

\section{Conclusions}

If new evidence supports reduction in the time to bedside standard, many more hospitals will not be adequately covered. Location-allocation optimisation is a powerful technique for supporting evidence-based service configuration. 


\section{Introduction}

Paediatric intensive care (PIC) services were centralised in the United Kingdom (UK) in 1997 (1). Dedicated regional PIC units (PICUs) were established, and specialist PIC retrieval teams (PICRTs) were established to transport critically ill children from district general hospitals (DGHs) to PICUs. PICRTs act as mobile intensive care teams: travelling to DGHs and commencing intensive care, ensuring that specialist expertise is not delayed until arrival at the PICU (2). Research has demonstrated that use of PICRTs (rather than non-specialist teams) for the transport of critically ill children improves the odds of their survival by $42 \%$ (3). The majority $(\sim 85 \%$,) of transports of critically ill children in the UK are performed by PICRTs (4).

Timely arrival at the patient's bedside is a key performance indicator for PICRTs and forms the basis of a current national quality standard which specifies that PICRTs should reach the patient bedside within 3 hours of accepting a referral (5). Data from the Paediatric Intensive Care Audit Network (PICANet) reveal that this standard is not always met by PICRTs (6), reflecting differences in mobilisation time (time from referral acceptance to departing the base) and/or journey time to the referring DGHs. As part of an ongoing national research study called DEPICT (7), we are assessing the impact that time taken by a PICRT to reach patient bedside has on clinical outcomes and patient experience in critically ill transported children. Findings from DEPICT will allow consideration of whether the 3-hour target should be increased or decreased, and whether a new service configuration is needed to meet any new standards.

One important aspect of PICRT configuration is how many PICRT locations are available and where PICRTs are located to minimise time to reach DGHs. The current location of PICRTs and the distribution of DGHs in their catchment area have evolved in ad hoc fashion based on historical referral patterns. In this paper, we use mathematical methods $(8,9)$ to explore, as a first step, how well current PICRT locations allow them to meet current, as well as any reduction in, national standards of time to bedside and the potential for different PICRT locations to reduce time to bedside. 


\section{Methods}

\section{Location-allocation modelling}

The question of where to locate "resource hubs" serving a number of "demand points" is perfectly suited to a branch of mathematics called "location-allocation optimisation" (8-11). In the context of this paper, the resource hubs are the PICRTs and the demand points are DGHs with acute paediatric services and also PICUs (in case of inter-PICU transfer). We explored scenarios where all PICUs could act as potential hosts for PICRTs and thus as potential resource hubs. Such models have been used to explore the location of emergency medical facilities (12-14) and general ambulances services (15). Location-allocation modelling can address questions such as: "What is the minimum number of PICRTs needed to reach all demand points within a specified period of time?" or "For a given number of PICRTs, where should they be located to minimise the journey time across all demand points and to which PICRT should each demand point be allocated?". The latter formulation is most relevant to the current situation in England and Wales: there are 11 current PICRTs, several hundred demand points and the PICS standard is 3 hours from referral acceptance to bedside. We can weight models by volume of demand from DGHs - so that hospitals that require PICRT services more are given greater priority in terms of minimising journey time to them from their allocated PICRT.

\section{Assumptions and parameters}

We assumed that a PICRT was always available to meet a referral, therefore time to bedside depended only on mobilisation time, journey time and time between the ambulance parking bay to the patient bedside at the DGH. We assumed a constant mobilisation time of 30 minutes (the PICS standard (16)) and a constant time of 10 minutes from arriving at the demand hospital to the child's bedside. Journey times were thus constrained to be 40 minutes less than the required time to bedside and only applied to transports performed by ground ambulances (not by air which comprise less than $2 \%$ of transports). In our analysis we assumed that journey times were constant between any 2 locations and we weighted our models by historic demand from each DGH.

\section{Questions we explored}

1. What proportion of overall demand can be covered from existing PICRTs for time to bedside standard of 3 hours and if standards are reduced following evidence from the DEPICT study? We consider times to bedside of 1 hour (20 minutes travel time), 75 minutes (35 minutes travel time), 90 minutes (50 minutes travel time), 2 hours (80 minutes travel time) and 3 hours (140 minutes travel time). 
2. What is the impact of reducing or increasing the number of PICRT locations on meeting different time to bedside standards assuming a) the current locations of PICRTs and b) assuming PICRTs could be located at any existing PICU or PICRT location?

\section{Definitions}

"Current locations" indicates that the mathematical model would be constrained to select PICRT locations from the pool of existing PICRTs if the number of PICRT locations is 11 or fewer. For more than 11 PICRTs, the model used the existing 11 PICRT locations first and could then choose additional PICRTs from any PICUs that are not already PICRTs. The "best locations" formulation allowed the model to choose PICRT locations from any existing PICU or PICRT location, regardless of whether a PICRT is currently based there or not.

The full mathematical formulation is provided in the Appendix.

\section{Travel time determination}

Distance and travel time between each PICRT, PICU and DGH was calculated using postcodes within the Google Maps Distance Matrix API (17) and the R package gmapsdistance (18). Google's "best guess" traffic model was used to estimate the travel time in minutes. We performed a sensitivity analysis of the estimated travel times by comparison with the "optimistic" and "pessimistic" traffic models - these did not change our results.

\section{Software}

The model was coded in Python using the library IBM Decision Optimization CPLEX Modelling for Python (19). Results were mapped using the Google Maps Geocoding API (20) and the Python module gmplot (21).

\section{Results}

\section{Location and journey time data}

The 24 PICUs and 11 PICRT teams in England and Wales are shown in Figure 1. We used a list of 212 DGHs with acute paediatric services and PICUs that generated demand for PICRT services at least once between 1 January and 31 December 2017 ( 5000 transports). The list of DGHs with acute paediatric services was obtained from the Royal College of Paediatrics and Child Health $(\mathrm{RCPCH})$ and the list of demand points for 2017 was obtained from PICANet. The demand from each demand hospital is shown in Figure 2. 


\section{Impact of different time to bedside thresholds using the 11 existing PICRT locations}

Figure 3 shows demand hospitals colour-coded by time to patient bedside for the current locations of 11 PICRTs after optimal allocation of demand hospitals to PICRTs (to minimise journey time). There are five hospitals not reachable within 3 hours (accounting for $1.3 \%$ of total demand): one in Cornwall, two in Wales and two in Norfolk.

For the current PICRT locations, 98\% of demand is reachable within 3 hours. Significant loss of coverage occurs if the standard is reduced to 2 hours, 90 minutes, or 75 minutes with $86 \%, 59 \%$, and $33 \%$ of demand reachable respectively. Less than $20 \%$ of the demand can be reached within an hour (20 minutes travel time).

\section{What is the impact of changing the number of PICRT locations on the demand reachable under different time to bedside standards?}

We examined the trade-off between the number of PICRT locations and the proportion of demand hospitals reachable within the five different 'time to bedside' time thresholds, using a) the current 11 PICRT locations as a starting point and b) any PICU as a potential PICRT location (Figure 4, solid and dashed lines respectively).

For a 3-hour threshold, there are only marginal gains in coverage above 8 PICRT locations (Figure 4). If the time standard is reduced to 2 hours however, then at least 13 PICRTs are needed to achieve a coverage of around $91 \%$ of demand (see the solid orange line in Figure 4). This requirement increases to 16, 21 and 24 PICRT locations for 90 minutes, 75 minutes and 1 hour respectively and with low achievable coverage (Figure 4).

Considering just 11 PICRT locations, could we improve on time to bedside by allowing them to be located at any of the currently unused PICUs? There is no meaningful difference for a 3 hour or 1-hour time to bedside threshold, but potentially substantial improvements for the other three thresholds: for 11 optimally located PICRTs, $91 \%$ of demand is reachable within 2 hours (compared to $86 \%$ now), $69 \%$ is reachable with 90 minutes (compared to $59 \%$ ), and $39 \%$ is reachable within 75 minutes (compared to $33 \%$ ).

The locations of the 11 optimally situated PICRTs and their allocated demand hospitals are shown in Figure 5 and compared to the current 11 PICRTs. Almost all the gains in proportion of demand covered are achieved in London, the South East and the Midlands. 


\section{Discussion}

The centralised model of PIC and transport has evolved over the past two decades largely based on historical referral patterns. This paper is the first attempt to use established mathematical methods to begin exploration of how current PICRT locations meet current national standards for 'time to bedside'.

Although not previously used in this setting, location-allocation optimisation methods have been used to model the optimal number and location of general ambulance services as well as emergency medical facilities. Daskin (12) created a model to meet $95 \%$ of demand within 10 minutes that also accounted for ambulance availability. Ruslim et al (22) noted that the mathematical models we used are particularly suitable for emergency services because they account for both travel time and population coverage criteria, meaning that higher demand hospitals are prioritised. Our study nonetheless had several limitations. Our models considered only the time to bedside of the PICRT to the DGH and not the time to destination PICU nor the time back to the PICRT base. Doing so may have had significant implications on our findings and conclusions. We used all demand for PICRTs in our model and did not distinguish between non-elective and elective transport. We treated mobilisation time and travel times as constant, and only considered existing PICUs as new potential PICRT locations. Importantly, we assumed that a PICRT team is always available when needed and did not account for known seasonal variation in demand from DGHs. However, addressing these limitations would act to increase time to bedside and thus our results represent optimistic estimates of coverage.

Under our assumptions, $98 \%$ of retrieval demand can be met within the 3-hour standard, with 5 DGHs not reachable by road within that time, making them candidates for the use of air transport. If the time to bedside standard was made more stringent, many fewer DGHs would be accessible within the standard. Recent analysis showed that the median PICRT mobilisation time was 29 minutes (IQR 17-65 minutes) (6). Reducing the PICRT mobilisation time (even by 15 minutes) could have a significant impact but would nonetheless be insufficient to meet most demand for any thresholds below 1.5 hours. Currently, $<2 \%$ of UK PICRT transports involve the use of rotary or fixed wing aircraft, mainly due to limited availability of aircraft for emergency inter-hospital transports. More stringent targets could start a national conversation about greater use of air transport or adding more PICRT locations to reduce road journey times.

Research has investigated the impact of distance (and thus, time) of the referring hospital to the admitting PICU. Ramnarayan et al (3) showed that there was a potential relationship 
between the distance to the admitting PICU and risk-adjusted mortality in the UK, with a greater odds ratio for patients transported from the most distant DGHs compared to those nearer (OR 1.42) although this was not statistically significant $(p=0.27)$.In a recent retrospective cohort study from Canada, Sample et al (23) found that remoteness of the referring hospital was associated with increased PICU length of stay, and PICU mortality was associated with duration of transport. These reports suggest that the time to PICU admission may indeed have some influence on patient outcomes; therefore, it is plausible that timeliness of access to a PICRT would have a similar impact. Findings from the DEPICT study will be crucial to inform further debate on this issue.

Our findings have several implications. First, there is an ongoing national review of paediatric critical care and transport services in England, which may suggest potential changes to PICRT configuration although there is currently little robust scientific evidence to support policy changes in this area. Our study shows that location-allocation optimisation is a powerful technique that can inform how services may be configured to deliver best outcomes for patients. Second, there is an increasing focus to deliver high quality care for retrieved patients and to continuously measure the performance of PICRTs while minimising cost. PICRTs are vital but expensive services to staff and maintain. The use of scientific methods to model how best to organise and deliver PICRTs is likely to form an important part of service development in the future. Third, the techniques used in this paper can be used to explore different scenarios as well as time to bedside targets and assess their impact on the location and number of PICRTs required, without embarking on any changes to the existing service structure. For instance, models can include varying the number of DGHs (if further consolidation of acute paediatric hospitals was predicted), varying the demand from each DGH (if activity was likely to change as a result of changes to population or consolidation of services) and varying the time to bedside target (if scientific evidence to indicate a beneficial effect of a reduced time to bedside became available), to explore the best service configuration. Models can also include other considerations such as team composition or diagnostic criteria in comparing different potential configuration designs.

Accounting for stochastic mobilisation and journey times is the next step in the mathematical modelling work stream of the DEPICT project. We will also extend the models by: using distributions of historical journey times reflecting the availability of "blue light" travel; incorporating queuing theory to take account of the likelihood of a transport team being available at referral (affecting mobilisation times) (see e.g. (12)); incorporating seasonal effects to capture the winter surge (see e.g. Ruslim's work on incorporating uncertain demand (22) and Pagel et al. (24) ); incorporating journey times for the rest of the transfer, 
that is time between local hospital to the receiving PICU and then time from the receiving PICU back to PICRT base (which affects availability of teams for subsequent transports).

The other work streams of the DEPICT study will provide a detailed assessment of factors affecting the clinical outcome of the child (e.g. underlying illness, age, treatments provided by the PICRT team, PICRT team composition) and those affecting parent and child experience of the transport (e.g. experience of waiting for a PICRT to arrive). Important factors identified in these strands of work will feed into the next stages of the mathematical modelling, so that we can explore the potential impact of e.g. changing team composition vs changing location configuration vs doing both or planning for seasonal teams, or more teams at some locations vs fewer teams at more locations.

Our initial results represent plausible "best case" scenarios of the allocation of demand hospitals to current PICRT services in England and Wales, made under simple starting assumptions. These add value by underscoring the importance of establishing an evidencebased time to bedside threshold, and other important factors affecting outcome, and then using existing, powerful, mathematical methods to inform the decision of where PICRTs could be located or otherwise configured. The current configuration can at best meet $98 \%$ of demand within the 3-hour time standard, leaving 5 hospitals not reachable by road within the 3-hour standard, but we would expect this coverage to be lower in real life with variable mobilisation and journey times, lack of teams during busy periods. This highlights the need for further study of the relation between the time standard and clinical outcomes before making changes to the PICRT service.

\section{Funding Acknowledgement}

The DEPICT project is funded by the National Institute for Health Research Health Services and Delivery Research programme (Project No: 15/136/45). The views and opinions expressed therein are those of the authors and do not necessarily reflect those of the NIHR HS\&DR programme or the Department of Health.

\section{DEPICT Study Group Acknowledgement}

The DEPICT study group members are: Elizabeth Draper; Jo Wray; Steve Morris; Patrick Davies; Will Marriage; Fatemah Rajah; Eithne Polke; Paul Mouncey; Anna Pearce; Matthew Entwistle; Rachel Lundy 


\section{What is known about this topic}

Current national standards expect a specialist paediatric intensive care retrieval team to reach the patient's bedside at the referring hospital within 3 hours, although this standard is not universally met.

There is an ongoing research study that will establish the impact of time to bedside on clinical outcomes for critically ill children and thus inform the national standard.

Mathematical location allocation modelling has been successfully used to research the location of emergency ambulance services.

\section{What this study adds}

This is the first mathematical evaluation of whether the current 3-hour standard for time to bedside is achievable for hospitals in England and Wales.

If research evidence supports more stringent time to bed standards, this would have significant implications for the configuration of paediatric intensive care retrieval team locations.

Mathematical location-allocation modelling has the potential to inform clinicians and commissioners regarding the optimal location of paediatric intensive care retrieval services. 


\section{References}

1. Department of Health. Paediatric Intensive Care "A framework for the future" [Internet]. 1997 [cited 2018 Jul 14]. Available from: http://www.ihrdni.org/315-016-1.pdf

2. Ramnarayan $P$, Polke $E$. The state of paediatric intensive care retrieval in Britain. Arch Dis Child. 2012 Feb;97(2):145-9.

3. Ramnarayan P, Thiru K, Parslow RC, Harrison DA, Draper ES, Rowan KM. Effect of specialist retrieval teams on outcomes in children admitted to paediatric intensive care units in England and Wales: a retrospective cohort study. Lancet Lond Engl. 2010 Aug 28;376(9742):698-704.

4. Annual report: Paediatric Intensive Care Audit Network [Internet]. HQIP. 2016 [cited 2018 Jul 14]. Available from: https://www.hqip.org.uk/resource/annual-report-paediatricintensive-care-audit-network/

5. Paediatric Intensive Care Society. Standards for the Care of Critically III Children [Internet]. 2010 [cited 2018 Jul 14]. Available from: http://picsociety.uk/wpcontent/uploads/2015/10/PICS_standards_2010.pdf

6. Ramnarayan P, Dimitriades K, Freeburn L, Kashyap A, Dixon M, Barry PW, et al. Interhospital Transport of Critically III Children to PICUs in the United Kingdom and Republic of Ireland: Analysis of an International Dataset. Pediatr Crit Care Med J Soc Crit Care Med World Fed Pediatr Intensive Crit Care Soc. 2018 Jun;19(6):e300-11.

7. Critically ill children and young people: do national Differences in access to Emergency Paediatric Intensive Care and care during Transport affect clinical outcomes and patient experience? The DEPICT study [Internet]. [cited 2018 Jul 30]. Available from: https://www.journalslibrary.nihr.ac.uk/programmes/hsdr/1513645/\#/

8. Smith HK, Laporte G, Harper PR. Locational analysis: highlights of growth to maturity. J Oper Res Soc. 2009 May 1;60(sup1):S140-8.

9. Batta R, Lejeune M, Prasad S. Public facility location using dispersion, population, and equity criteria. Eur J Oper Res. 2014 May 1;234(3):819-29.

10. Hakimi SL. Optimum Locations of Switching Centers and the Absolute Centers and Medians of a Graph. Oper Res. 1964 Jun 1;12(3):450-9.

11. Hakimi SL. Optimum Distribution of Switching Centers in a Communication Network and Some Related Graph Theoretic Problems. Oper Res. 1965 Jun 1;13(3):462-75.

12. Daskin MS. Network and Discrete Location: Models, Algorithms, and Applications. 2nd Revised edition edition. Hoboken, New Jersey: Wiley-Blackwell; 2013. 536 p.

13. Coskun N, Erol R. An Optimization Model for Locating and Sizing Emergency Medical Service Stations. J Med Syst. 2010 Feb 1;34(1):43-9.

14. Toregas C, Swain R, ReVelle C, Bergman L. The Location of Emergency Service Facilities. Oper Res. 1971;19(6):1363-73.

15. Brotcorne L, Laporte G, Semet F. Ambulance location and relocation models. Eur J Oper Res. 2003 Jun 16;147(3):451-63. 
16. Paediatric Intensive Care Society. PICU Quality Dashboard 2017/18 [Internet]. [cited 2018 Aug 13]. Available from: https://www.england.nhs.uk/wpcontent/uploads/2017/08/paediatric-intensive-care-unit-metric-definitions-2017-18.pdf

17. Developer Guide | Distance Matrix API [Internet]. Google Developers. [cited 2018 Jul 23]. Available from: https://developers.google.com/maps/documentation/distancematrix/intro

18. Rodrigo Azuero Melo \& \& Demetrio Rodriguez T \& David Zarruk (2018). gmapsdistance: Distance and Travel Time Between Two Points from Google Maps. R package version 3.4. https://CRAN.R-project.org/package=gmapsdistance

19. IBM $\circledast$ Decision Optimization CPLEX® Modeling for Python - IBM® Decision Optimization CPLEX® Modeling for Python (DOcplex) V2.7 documentation [Internet]. [cited $2018 \mathrm{Jul}$ 23]. Available from: http://ibmdecisionoptimization.github.io/docplexdoc/index.html

20. Developer Guide | Geocoding API [Internet]. Google Developers. [cited 2018 Jul 23]. Available from: https://developers.google.com/maps/documentation/geocoding/intro

21. Woods M. gmplot: Plotting data on google maps, the easy (stupid) way [Internet]. 2018. Available from: https://github.com/vgm64/gmplot

22. Mohd Ruslim N, Ghani N. An Application of the p-Median Problem with Uncertainty in Demand in Emergency Medical Services. 2006 Jan 1;

23. Sample M, Acharya A, O'Hearn K, Livingstone S, Menon K. The Relationship Between Remoteness and Outcomes in Critically III Children. Pediatr Crit Care Med J Soc Crit Care Med World Fed Pediatr Intensive Crit Care Soc. 2017 Nov;18(11):e514-20.

24. Pagel C, Ramnarayan P, Ray S, Peters MJ. A Novel Method to Identify the Start and End of the Winter Surge in Demand for Pediatric Intensive Care in Real Time. Pediatr Crit Care Med J Soc Crit Care Med World Fed Pediatr Intensive Crit Care Soc. 2015 Nov;16(9):821-7.

25. Li X, Zhao Z, Zhu X, Wyatt T. Covering models and optimization techniques for emergency response facility location and planning: a review. Math Methods Oper Res. 2011 Dec 1;74(3):281-310. 
Figures

Figure 1- Map of PICUs (red) and PICRTs (blue). Expanded sections of the map for Newcastle and London are shown on the right hand side. An HTML file showing this information as an interactive Google Map can be downloaded from https://figshare.com/s/e2ad394365fcb456b5cd (doi: 10.6084/m9.figshare.7611659) and viewed in any internet browser. The HTML map is zoomable and scrollable and the hospitals are labelled.

Figure 2 - Map of demand at DGHs, by quintile of demand for PICRT transports during 2017. An HTML file showing this information as an interactive Google Map can be downloaded from https://figshare.com/s/e2ad394365fcb456b5cd (doi: 10.6084/m9.figshare.7611659) and viewed in any internet browser. The HTML map is zoomable and scrollable and the hospitals are labelled with name and annual demand for 2017.

Figure 3 - Demand hospitals colour-coded by time to bedside with the current configuration of PICRTs (light blue). Demand hospitals reachable within 1 hour, 75 minutes, 90 minutes, 2 hours and 3 hours of their assigned PICRT are coloured blue, green, yellow, orange, and red respectively. Black markers indicate hospitals not reachable within 3 hours. An HTML file showing this information as an interactive Google Map can be downloaded from https://figshare.com/s/e2ad394365fcb456b5cd (doi: 10.6084/m9.figshare.7611659) and viewed in any internet browser. The HTML map is zoomable and scrollable; hospitals are labelled with name, annual demand for 2017 and allocated PICRT.

Figure 4 - Percent of demand covered with time to bedside 1-3 hours for $3-27$ current and optimal PICRT locations and optimal demand hospital allocation. Black vertical line indicates the coverage for 11 PICRT locations. Solid and dashed lines represent the current and best locations respectively.

Figure 5 - Optimal allocation of DGHs to current and best locations of 11 PICRTs. Black markers indicate hospitals not reachable within 3 hours of a PICRT or PICU. An HTML file showing this information as an interactive Google Map can be downloaded from https://figshare.com/s/e2ad394365fcb456b5cd (doi: 10.6084/m9.figshare.7611659) and viewed in any internet browser. The HTML map is zoomable and scrollable; hospitals are labelled with name, annual demand for 2017 and allocated PICRT. 